\title{
Programas de Controle de Infecção Relacionada à Assistência à Saúde: avaliação de indicadores de estrutura e processo
}

\author{
Infection Control Programs Related to Healthcare: evaluation of structure and process indicators \\ Programas de Control de Infecciones Relacionados con la Salud: evaluación de indicadores de
}

estructura y proceso

Recebido: 08/12/2021 | Revisado: 15/12/2021 | Aceito: 24/12/2021 | Publicado: 04/01/2022

\author{
Ione Rocha Neves \\ ORCID: https://orcid.org/0000-0002-9051-0762 \\ Faculdade São Leopoldo Mandic, Brasil \\ E-mail: yone.neves@hotmail.com \\ Flávia Martão Flório \\ ORCID: https://orcid.org/0000-0001-7742-0255 \\ Faculdade São Leopoldo Mandic, Brasil \\ E-mail: flaviaflorio@yahoo.com.br \\ Luciane Zanin \\ ORCID: http://orcid.org/0000-0003-0218-9313 \\ Faculdade São Leopoldo Mandic, Brasil \\ E-mail: zaninsouza@yahoo.com.br
}

\begin{abstract}
Resumo
Este estudo possui o objetivo de avaliar os Programas de Controle de Infecção Relacionada à Assistência à Saúde em oito hospitais municipais de São Luís - MA. Trata-se de estudo descritivo e exploratório, quantitativo, realizado em 2020, por meio de aplicação de um instrumento constituído por quatro indicadores clínicos, sendo dois de estrutura (Estrutura Técnico-Operacional do Programa de Controle de Infecção Relacionada à Assistência à Saúde e Diretrizes Operacionais de Controle e Prevenção de Infecção Relacionada à Assistência à Saúde) e dois de processos (Sistema de Vigilância Epidemiológica de Infecção Relacionada à Assistência à Saúde e Atividades de Controle e Prevenção de Infecção Relacionada à Assistência à Saúde). $\mathrm{O}$ indicador que avalia as diretrizes operacionais apresentou a maior média de conformidade $(70,5 \%)$, seguido do indicador que avalia a estrutura técnico-operacional $(68,8 \%)$. Os indicadores de estrutura apresentaram menor conformidade (37,5\%) nos componentes reuniões com lideranças e gestores, espaço físico exclusivo para suas atividades e padronização de soluções germicidas e antissépticos. Os indicadores de processo apresentaram menores índices nos componentes relacionados à realização de relatórios relativos às infecções hospitalares $(37,5 \%)$ e realização de atividades de controle e prevenção no setor de nutrição e dietética $(12,5 \%)$. Os serviços avaliados apresentam melhor desempenho nos indicadores de estrutura e denotam fragilidade quanto às atividades essenciais para prevenção de infecções hospitalares, como realização de vigilância epidemiológica e desenvolvimento de estratégias de prevenção e controle destas infecções em diversos setores do hospital.
\end{abstract}

Palavras-chave: Infecção hospitalar; Programa de controle de infecção hospitalar; Avaliação de serviços de saúde.

\begin{abstract}
This study aims to evaluate the Health Care-Related Infection Control Programs in eight municipal hospitals in São Luís - MA. This is a descriptive and exploratory, quantitative study, carried out in 2020, through the application of an instrument consisting of four clinical indicators, two of which are structural (Technical-Operational Structure of the Health Care-Related Infection Control Program and Guidelines Operational Control and Prevention of Infection Related to Health Care) and two of processes (Epidemiological Surveillance System for Infection Related to Health Care and Control and Prevention Activities Related to Health Care). The indicator that assesses the operational guidelines had the highest average of compliance $(70.5 \%)$, followed by the indicator that assesses the technicaloperational structure (68.8\%). The structure indicators showed lower compliance (37.5\%) in the components meetings with leaders and managers, exclusive physical space for their activities and standardization of germicidal and antiseptic solutions. Process indicators had lower rates in the components related to reporting on hospital infections (37.5\%) and carrying out control and prevention activities in the nutrition and diet sector (12.5\%). The services evaluated show better performance in the structure indicators and show fragility regarding essential activities for the prevention of hospital infections, such as carrying out epidemiological surveillance and developing prevention and control strategies for these infections in different sectors of the hospital.
\end{abstract}

Keywords: Hospital infection; Hospital infection control program; Health service assessment. 


\begin{abstract}
Resumen
Este estudio tiene como objetivo evaluar los Programas de Control de Infecciones Relacionadas con la Atención de la Salud en ocho hospitales municipales de São Luís - MA. Se trata de un estudio descriptivo y exploratorio, cuantitativo, realizado en el año 2020, mediante la aplicación de un instrumento compuesto por cuatro indicadores clínicos, dos de los cuales son estructurales (Estructura Técnico-Operativa del Programa de Control de Infecciones Relacionadas con la Salud y Lineamientos de Control Operacional y Prevención de Infecciones Relacionadas con la Atención de la Salud) y dos de procesos (Sistema de Vigilancia Epidemiológica de Infecciones Relacionadas con la Atención de la Salud y Actividades de Control y Prevención Relacionadas con la Atención de la Salud). El indicador que evalúa los lineamientos operativos presentó el mayor promedio de cumplimiento $(70,5 \%)$, seguido del indicador que evalúa la estructura técnico-operativa $(68,8 \%)$. Los indicadores de estructura mostraron menor cumplimiento (37,5\%) en las reuniones de componentes con líderes y gerentes, espacio físico exclusivo para sus actividades y estandarización de soluciones germicidas y antisépticas. Los indicadores de proceso presentaron menores tasas en los componentes relacionados con la notificación de infecciones hospitalarias $(37,5 \%)$ y la realización de actividades de control y prevención en el sector de nutrición y dieta (12,5\%). Los servicios evaluados muestran un mejor desempeño en los indicadores de estructura y muestran fragilidad en las actividades esenciales para la prevención de infecciones hospitalarias, como la realización de vigilancia epidemiológica y el desarrollo de estrategias de prevención y control de estas infecciones en diferentes sectores del hospital.
\end{abstract}

Palabras clave: Infección hospitalaria; Programa de control de infecciones hospitalarias; Evaluación de servicios de salud.

\title{
1. Introdução
}

Infecção hospitalar é aquela adquirida após a admissão do paciente e que se manifesta durante a internação ou após a alta, quando puder ser relacionada com a internação ou procedimentos hospitalares (Brasil, 1998). Também pode ser tratada como Infecção Relacionada à Assistência à Saúde (IRAS) por ter sido reconhecido que a temática é complexa e multifatorial, indo além do ambiente hospitalar (Siegel et al., 2007; Bordignon et al., 2020).

As IRAS são um problema de magnitude global, que suscitam crescente preocupação entre profissionais de saúde, administradores de instituições prestadoras de assistência e formuladores de políticas públicas de saúde (Schmitt, 2015). Estas infecções estão relacionadas a um alto índice de morbimortalidade e estão entre as principais complicações após internação, podendo ocasionar afastamento do paciente de suas tarefas profissionais e familiares, além do impacto socioeconômico causado, visto que podem resultar em novos procedimentos terapêuticos de elevado custo, aumentando o tempo de hospitalização (Costa, 2016; Instituto Latino Americano de Sepse, 2016; Storr et al., 2017). Ainda assim, estima-se que a cada 100 pacientes hospitalizados, sete em países desenvolvidos e 10 em países em desenvolvimento irão adquirir pelo menos uma infecção hospitalar (World Health Organization, 2014).

Considerando que as infecções hospitalares constituem risco significativo à saúde, a legislação brasileira instituiu, para todos os hospitais do Brasil, a implementação de Programas de Controle de Infecções Relacionadas à Assistência à Saúde (PCIRAS) e a constituição de Comissões de Controle de Infecção Hospitalar (CCIH), que devem atuar de forma eficiente, visando um adequado controle dessas infecções (Brasil, 1997; Brasil, 1998). Desde que foram criados, as principais avaliações dos PCIRAS se reduzem predominantemente a descrição de taxas de infecções hospitalares apresentadas na instituição (Alves \& Lacerda, 2015). De acordo com Oliveira, Silva e Lacerda (2016), ainda que as atividades dos PCIRAS acompanhem a evolução tecnológica e as evidências científicas das práticas assistenciais, em muitas instituições o processo de trabalho permanece semelhante desde sua origem, pautado predominantemente em indicadores epidemiológicos de resultados de ocorrência dessas infeções. Isso faz com que esta forma de avaliação seja insuficiente para reconhecer outros aspectos relacionados à estrutura e ao processo de trabalho desses programas que podem comprometer a segurança dos procedimentos realizados na instituição (Alves \& Lacerda, 2015).

No Brasil, estudos têm sido realizados utilizando indicadores clínicos para avaliação de PCIRAS, como realizado no estado do Paraná, onde o indicador que avalia a estrutura técnico-operacional apresentou maior média de conformidade (79,4\%), e no município de Campo Grande, Mato Grosso do Sul, onde os melhores resultados foram evidenciados nos 
indicadores que avaliam a estrutura técnico-operacional e a vigilância epidemiológica de IRAS, com médias de conformidade de $80,58 \%$ e $81,59 \%$, respectivamente (Alves \& Lacerda, 2015; Giroti et al., 2018).

Estudos desta magnitude, ainda não foram realizados no município de São Luís, no Maranhão, onde a temática infecção hospitalar tem sido contemplada em pesquisas com diversas abordagens, porém todas diferem da aqui apresentada. Com o intuito de contribuir para uma melhor qualificação das práticas de controle de infecção em hospitais municipais de São Luís, este estudo teve o objetivo de avaliar os Programas de Controle de Infecção Relacionada à Assistência à Saúde em oito hospitais municipais de São Luís - MA.

\section{Metodologia}

Trata-se de um estudo descritivo e exploratório, com abordagem quantitativa realizado na capital do estado do Maranhão, São Luís, que possui área territorial de $582,974 \mathrm{~km}^{2}$, população de $1.101,884$ habitantes e Índice de Desenvolvimento Humano de 0,768 (Instituto Brasileiro de Geografia e Estatística, 2010).

Inicialmente foram verificados, por meio do Cadastro Nacional de Estabelecimentos de Saúde (CNES), todos os hospitais públicos com gestão municipal, geral ou especializado, com leitos de internação, e que possuam como entidade mantenedora a Secretaria Municipal de Saúde. Do total de 81 instituições, foram excluídas 73 unidades de saúde, sendo 60 destinadas a cuidados exclusivamente ambulatoriais, cinco a atendimentos psiquiátricos, uma maternidade, três centros de especialidades odontológicas, duas centrais de marcação de consultas e exames, um centro de controle de zoonoses e um laboratório central.

Desta forma, a amostra foi composta por oito hospitais da rede municipal, sendo um hospital especializado no atendimento à mulher, um hospital pediátrico e seis hospitais de urgência e emergência, sendo que entre estes, quatro dispõem de pronto atendimento apenas nas especialidades de clínica médica e pediátrica.

Como instrumento de coleta de dados, foram utilizados quatro indicadores clínicos, previamente construídos e validados por Silva e Lacerda (2007), para avaliação de PCIRAS, sendo dois relacionados à estrutura e dois relacionados ao processo de trabalho, os quais abordavam as seguintes questões:

1. Estrutura Técnico-Operacional do PCIRAS (indicador de estrutura) - avalia se a CCIH é composta por, no mínimo, membros executores (sendo um deles, um enfermeiro, que atua 6 horas por dia, e outro profissional de nível superior, que atue 4 horas por dia) e membros consultores, representantes do serviço médico, enfermagem e administração; possui regimento interno; realiza reuniões periódicas; tem suporte de laboratório de microbiologia e patologia; possui espaço físico delimitado e exclusivo, com recursos informatizados, para as atividades diárias e se a administração disponibiliza dados estatísticos para realização de relatórios.

2. Diretrizes Operacionais de Controle e Prevenção de IRAS (indicador de estrutura) - avalia se há recomendações para: avaliação e encaminhamento de acidentes com exposições a material biológico; descarte de resíduos; controle e prevenção de IRAS, isolamento de pacientes; uso de antibióticos profiláticos; padronização de soluções germicidas e antissépticos; técnica de limpeza e desinfecção de materiais, equipamentos e superfícies; higienização de mãos; lavagem de roupas da instituição; coleta de material para culturas e realização de curativos.

3. Sistema de Vigilância Epidemiológica de IRAS (indicador de processo) - avalia se a CCIH realiza periodicamente a vigilância epidemiológica das IRAS, por meio de busca ativa de casos e em unidades de maior risco, como Unidades de Terapia Intensiva (UTI); monitora os resultados microbiológicos de culturas; possui critérios prédeterminados para diagnóstico de IRAS; produz, e disponibiliza para setores, lideranças e órgãos públicos concernentes, relatórios periódicos dos resultados da vigilância epidemiológica, informando as alterações do perfil epidemiológico e correlacionando resultados com intervenções adotadas. 
4. Avaliação das Atividades de Controle e Prevenção de IRAS (indicador de processo) - avalia se a CCIH participa nas decisões técnicas para especificação e aquisição de produtos e correlatos, e realiza visita para avaliação, orientação de melhorias técnicas e adequações do ambiente em Unidades de Diálise, Banco de Sangue, Laboratórios de Análises Clínicas e Anatomia Patológica, Unidades de Internação, UTI, Berçário, Centro Cirúrgico, Central de Material e Esterilização, Pronto Socorro, Ambulatório, Farmácia e Serviço de Nutrição e Dietética.

Cada item avaliado foi classificado pelo pesquisador para posterior cálculo dos índices de conformidade considerando no numerador o total de práticas em conformidade e no denominador o total de práticas avaliadas. A conformidade total foi considerada quando atendeu completamente o item avaliado, a não conformidade foi considerada quando o item avaliado não foi identificado e a conformidade parcial foi considerada quando os serviços de saúde apresentaram apenas parte da documentação exigida. $\mathrm{O}$ critério não se aplica foi considerado quando o serviço não apresentou o item sob avaliação por não fazer parte do seu quadro de procedimentos exigidos para aquele serviço.

A coleta de dados foi realizada em novembro e dezembro de 2020, pela própria pesquisadora, por meio de avaliação de documentos solicitados no instrumento de pesquisa (normas institucionais, atas de instituição, atas de reuniões, relatórios ou qualquer outro registo formal utilizado no serviço de saúde) e entrevista presencial com um membro executor responsável pela CCIH. Em todos os hospitais, o profissional entrevistado referiu-se ao enfermeiro, que concordou em participar da pesquisa, mediante assinatura em Termo de Consentimento Livre e Esclarecido. Em duas unidades a coleta dos dados foi realizada na primeira visita realizada no hospital; nas demais, foi agendado um segundo momento para a coleta dos mesmos.

Os dados foram armazenados em banco de dados, e analisados de forma descritiva por meio de frequências absolutas e relativas. A pesquisa foi aprovada pelo Comitê de Ética em Pesquisa (CEP) da Faculdade São Leopoldo Mandic - Campinas - SP, CAAE nº 35597020.0.0000.5374.

\section{Resultados}

Entre os oito hospitais que participaram do estudo, 50\% eram unidades consideradas de pequeno porte, e as demais, de médio (25\%) e de grande porte (25\%). Quatro possuíam UTI, Banco de Sangue e Laboratório de Análises Clínicas; três possuíam Centro Cirúrgico e apenas duas, Unidades de Diálise. Nenhuma possuía Laboratório de Anatomia Patológica e Bercário. Em todas as unidades havia $\mathrm{CCIH}$ próprias, não consorciadas, com tempo de funcionamento superior a um ano.

Ao considerar a conformidade de cada componente dos quatro indicadores avaliados verificou-se que a média de conformidade geral foi de 60,0\%. Em relação ao indicador Estrutura Técnico-Operacional do PCIRAS, que apresentou média de conformidade de $68,8 \%$ (com desvio padrão de $22,2 \%$ ), os componentes que avaliam a CCIH quanto à realização de reuniões periódicas com participação de lideranças do hospital e presença de espaço físico exclusivo para realização das atividades diárias, obtiveram menores índices de conformidade, 37,5\% (Tabela 1). 
Tabela 1. Distribuição das conformidades por componentes do indicador "Estrutura Técnico-Operacional do PCIRAS" em hospitais municipais de São Luís - MA, Brasil, 2020.

\begin{tabular}{|c|c|c|c|c|c|c|c|}
\hline \multirow{3}{*}{ Componentes } & \multirow{3}{*}{$\begin{array}{c}\text { Aplicável } \\
\text { n }\end{array}$} & \multicolumn{6}{|c|}{ Conformidade } \\
\hline & & \multicolumn{2}{|c|}{ Sim } & \multicolumn{2}{|c|}{ Não } & \multicolumn{2}{|c|}{ Parcial } \\
\hline & & $\mathbf{n}$ & $\%$ & $\mathbf{n}$ & $\%$ & $\mathbf{n}$ & $\%$ \\
\hline $\begin{array}{l}\text { A CCIH é representada, no mínimo, por membros consultores do serviço } \\
\text { médico, enfermagem e administração. }\end{array}$ & 8 & 7 & 87,5 & 1 & 12,5 & - & - \\
\hline Há um regimento que determina o funcionamento da CCIH. & 8 & 7 & 87,5 & 1 & 12,5 & - & - \\
\hline Há 2 membros executores, de nível superior, sendo um, enfermeiro. & 8 & 4 & 50,0 & 4 & 50,0 & - & - \\
\hline O enfermeiro atua 6 horas/dia, com dedicação exclusiva. & 8 & 7 & 87,5 & 1 & 12,5 & - & - \\
\hline Há outro profissional, com nível superior, que atua 4 horas/dia. & 8 & 4 & 50,0 & 4 & 50,0 & - & - \\
\hline A CCIH realiza reuniões periódicas com lideranças do hospital. & 8 & 3 & 37,5 & 5 & 62,5 & - & - \\
\hline Há suporte de laboratório de microbiologia e patologia. & 8 & 7 & 87,5 & 1 & 12,5 & - & - \\
\hline Há espaço físico exclusivo para as atividades diárias da CCIH. & 8 & 3 & 37,5 & 5 & 62,5 & - & - \\
\hline Há disponibilização de recursos informatizados para a CCIH. & 8 & 7 & 87,5 & 1 & 12,5 & - & - \\
\hline Há disponibilização de dados estatísticos para realizar os relatórios. & 8 & 6 & 75,0 & 2 & 25,0 & - & - \\
\hline Média & & & 68,8 & & 31,2 & & - \\
\hline Desvio padrão & & & 22,2 & & 22,2 & & - \\
\hline
\end{tabular}

Fonte: Autores (2021).

O indicador que avalia as diretrizes operacionais de controle e prevenção de IRAS, apresentou média de conformidade de 70,5\%, com desvio padrão de 19,9\%. O item "Há padronização de soluções germicidas e antissépticos" obteve o menor índice de conformidade, $37,5 \%$, sendo que o maior índice, 100\%, foi apresentado pelos componentes "Há recomendações para controle e prevenção de infecções de sítio cirúrgico" e "Há recomendações para o uso de antibióticos para prevenção de infecção de sítio cirúrgico", ambos aplicáveis em apenas três instituições. O segundo maior índice, 87,5\%, referiu-se aos componentes "Há recomendações para isolamentos de pacientes com doenças infectocontagiosas ou imunodeprimidos", "Há recomendações para controle e prevenção de infecções urinárias" e "Há recomendação para técnica de higienização de mãos" (Tabela 2).

Tabela 2. Distribuição das conformidades por componentes do indicador "Diretrizes Operacionais de Controle e Prevenção de IRAS”, quanto ao registro de recomendações, em hospitais municipais de São Luís - MA, Brasil, 2020.

\begin{tabular}{|c|c|c|c|c|c|c|c|}
\hline \multirow{3}{*}{ Componentes } & \multirow{3}{*}{$\begin{array}{c}\text { Aplicável } \\
\text { n* }\end{array}$} & \multicolumn{6}{|c|}{ Conformidade } \\
\hline & & \multicolumn{2}{|c|}{ Sim } & \multicolumn{2}{|c|}{ Não } & \multicolumn{2}{|c|}{ Parcial } \\
\hline & & $\mathbf{n}$ & $\%$ & $\mathbf{n}$ & $\%$ & $\mathbf{n}$ & $\%$ \\
\hline $\begin{array}{l}\text { Há recomendação para avaliação e encaminhamento de acidentes com } \\
\text { exposições a material biológico. }\end{array}$ & 8 & 5 & 62,5 & 3 & 37,5 & - & - \\
\hline Para descarte de resíduos de serviço de saúde. & 8 & 5 & 62,5 & 3 & 37,5 & - & - \\
\hline Para controle e prevenção de infecções respiratórias. & 8 & 6 & 75,0 & 2 & 25,0 & - & - \\
\hline Para controle e prevenção de infecções urinárias. & 8 & 7 & 87,5 & 1 & 12,5 & - & - \\
\hline Para prevenção de infecções de corrente sanguínea. & 8 & 6 & 75,0 & 2 & 25,0 & - & - \\
\hline Para controle e prevenção de infecções de sítio cirúrgico. & 3 & 3 & 100,0 & - & - & - & - \\
\hline $\begin{array}{l}\begin{array}{l}\text { Para isolamentos de pacientes com doenças infectocontagiosas ou } \\
\text { imunodeprimidos. }\end{array} \\
\end{array}$ & 8 & 7 & 87,5 & 1 & 12,5 & - & - \\
\hline Para uso de antibióticos para prevenção de infecção de sítio cirúrgico. & 3 & 3 & 100,0 & - & - & - & - \\
\hline $\begin{array}{l}\text { Para técnicas de limpeza, desinfecção e esterilização de materiais e } \\
\text { equipamentos. }\end{array}$ & 8 & 4 & 50,0 & 4 & 50,0 & - & - \\
\hline Para técnica de higienização de mãos. & 8 & 7 & 87,5 & 1 & 12,5 & - & - \\
\hline Para rotina de limpeza e desinfecção de superfícies. & 8 & 5 & 62,5 & 3 & 37,5 & - & - \\
\hline Para lavagem e higienização de roupas utilizadas na instituição. & - & - & - & - & - & - & - \\
\hline Para coleta de material para realização de culturas. & 8 & 4 & 50,0 & 4 & 50,0 & - & - \\
\hline Para técnicas de curativos e periodicidade de trocas dos mesmos. & 8 & 4 & 50,0 & 4 & 50,0 & - & - \\
\hline Há padronização de soluções germicidas e antissépticos. & 8 & 3 & 37,5 & 5 & 62,5 & - & - \\
\hline Média & & & 70,5 & & 29,5 & & - \\
\hline Desvio padrão & & & 19,9 & & 19,9 & & - \\
\hline
\end{tabular}

*O n apresentou variação entre os componentes, pois alguns não eram aplicáveis a todos os hospitais participantes. Fonte: Autores (2021). 
O indicador Sistema de Vigilância Epidemiológica de IRAS, que apresentou média de conformidade 55,7\%, com desvio padrão de 20,5\%, mostrou o menor índice, 37,5\%, em relação aos componentes que avaliam se os relatórios periódicos informam alterações do perfil epidemiológico, correlacionam os resultados com estratégias a serem adotadas, e são regularmente disponibilizados aos diversos setores do hospital, lideranças da instituição e órgãos públicos concernentes. O componente que avalia se a CCIH realiza busca ativa de casos de IRAS nas unidades de maior risco, como UTI, aplicável em apenas quatro instituições, obteve a taxa mais alta de conformidade, 100\% (Tabela 3).

Tabela 3. Distribuição das conformidades por componentes do indicador "Sistema de Vigilância Epidemiológica de IRAS" em hospitais municipais de São Luís - MA, Brasil, 2020.

\begin{tabular}{|c|c|c|c|c|c|c|c|}
\hline \multirow{3}{*}{ Componentes } & \multirow{3}{*}{$\begin{array}{c}\text { Aplicável } \\
\mathbf{n}^{*}\end{array}$} & \multicolumn{6}{|c|}{ Conformidade } \\
\hline & & \multicolumn{2}{|c|}{ Sim } & \multicolumn{2}{|c|}{ Não } & \multicolumn{2}{|c|}{ Parcial } \\
\hline & & $\mathbf{n}$ & $\%$ & $\mathbf{n}$ & $\%$ & $\mathbf{n}$ & $\%$ \\
\hline Realiza vigilância epidemiológica com periodicidade determinada. & 8 & 6 & 75,0 & 2 & 25,0 & - & - \\
\hline Realiza vigilância epidemiológica de IRAS através de busca ativa. & 8 & 5 & 62,5 & 3 & 37,5 & - & - \\
\hline Realiza busca ativa de casos de IRAS nas unidades de maior risco. & 4 & 4 & 100,0 & - & - & - & - \\
\hline Monitora regularmente resultados microbiológicos de culturas. & 7 & 4 & 57,1 & 3 & 42,9 & - & - \\
\hline Há critérios pré-determinados para diagnóstico de IRAS. & 8 & 5 & 62,5 & 3 & 37,5 & - & - \\
\hline Produz relatório periódico dos resultados da vigilância. & 8 & 4 & 50,0 & 4 & 50,0 & - & - \\
\hline Os relatórios analisam as alterações do perfil epidemiológico. & 8 & 3 & 37,5 & 5 & 62,5 & - & - \\
\hline Os relatórios correlacionam resultados com estratégias adotadas. & 8 & 3 & 37,5 & 5 & 62,5 & - & - \\
\hline Os relatórios são disponibilizados a outros setores da instituição. & 8 & 3 & 37,5 & 5 & 62,5 & - & - \\
\hline Os relatórios são disponibilizados para os órgãos públicos & 8 & 3 & 37,5 & 5 & 62,5 & - & - \\
\hline Média & & & 55,7 & & 44,3 & & - \\
\hline Desvio padrão & & & 20,5 & & 20,5 & & - \\
\hline
\end{tabular}

*O n apresentou variação entre os componentes, pois alguns não eram aplicáveis a todos os hospitais participantes. Fonte: Autores (2021).

O indicador Avaliação das Atividades de Controle e Prevenção de IRAS apresentou menor média de conformidade $(44,9 \%)$ entre os quatro indicadores, com desvio padrão de $34,7 \%$. Os componentes apresentaram conformidades que variaram de $12,5 \%$ a $100 \%$, sendo o maior índice, $100 \%$, referente aos componentes que avaliam a realização destas atividades em unidades de internação e UTI, e no componente que avalia a participação dos controladores de infecção nas decisões técnicas para especificação e aquisição de produtos e correlatos (aplicável em apenas uma instituição). A menor conformidade foi identificada no componente que avalia a realização destas atividades no Serviço de Nutrição e Dietética (Tabela 4). 
Tabela 4. Distribuição das conformidades por componentes do indicador "Atividades de Controle e Prevenção de IRAS", quanto às atividades de controle e prevenção* desenvolvidas por setor e quanto à participação em decisões para aquisições de produtos e correlatos, em hospitais municipais de São Luís - MA, Brasil, 2020.

\begin{tabular}{|c|c|c|c|c|c|c|c|}
\hline \multirow{3}{*}{ Componentes } & \multirow{3}{*}{$\begin{array}{c}\text { Aplicável } \\
\mathrm{n}^{*}\end{array}$} & \multicolumn{6}{|c|}{ Conformidade } \\
\hline & & \multicolumn{2}{|r|}{ Sim } & \multicolumn{2}{|c|}{ Não } & \multicolumn{2}{|c|}{ Parcial } \\
\hline & & $\mathbf{n}$ & $\%$ & $\mathbf{n}$ & $\%$ & $\mathbf{n}$ & $\%$ \\
\hline Unidade de Diálise. & 2 & 1 & 50,0 & - & - & 1 & 50,0 \\
\hline Banco de Sangue. & 4 & 1 & 25,0 & 2 & 50,0 & 1 & 25,0 \\
\hline Laboratório de Anatomia Patológica. & - & - & - & - & - & - & - \\
\hline Laboratório de Análises Clínicas. & 4 & 1 & 25,0 & 1 & 25,0 & 2 & 50,0 \\
\hline Unidade de Internação. & 8 & 8 & 100,0 & - & - & - & - \\
\hline Unidade de Terapia Intensiva & 4 & 4 & 100,0 & - & - & - & - \\
\hline Berçário & - & - & - & - & - & - & - \\
\hline Central de Material e Esterilização & 8 & 3 & 37,5 & 3 & 37,5 & 2 & 25,0 \\
\hline Centro Cirúrgico. & 3 & 1 & 33,3 & 1 & 33,3 & 1 & 33,3 \\
\hline Pronto Socorro. & 7 & 1 & 14,3 & - & - & 6 & 85,7 \\
\hline Ambulatório. & 6 & 1 & 16,7 & 2 & 33,3 & 3 & 50,0 \\
\hline Serviço de Farmácia. & 8 & 2 & 25,0 & 2 & 25,0 & 4 & 50,0 \\
\hline Serviço de Nutrição e Dietética. & 8 & 1 & 12,5 & 1 & 12.5 & 6 & 75,0 \\
\hline $\begin{array}{l}\text { Participa nas decisões técnicas para especificação } \\
\text { e aquisição de produtos e correlatos. }\end{array}$ & 1 & 1 & 100,0 & - & - & - & - \\
\hline Média & & & 44,9 & & 18,5 & & 37,1 \\
\hline Desvio padrão & & & 34,7 & & 18,9 & & 28,4 \\
\hline
\end{tabular}

*As atividades de controle de prevenção de IRAS compreendem visitas de inspeção previamente programadas, orientações e avaliações de diretrizes introduzidas, participação em reuniões dos setores, realização de consultas e esclarecimentos cotidianos por ocasião de demandas espontâneas.

**O n apresentou variação entre os itens avaliados, pois alguns deles não eram aplicáveis a todos os hospitais participantes. Fonte: Autores (2021).

O componente "Participa nas decisões técnicas para especificação e aquisição de produtos e correlatos", foi considerado inaplicável em sete instituições, pois nestas, as decisões técnicas para aquisições de produtos são tomadas diretamente pela Secretaria Municipal de Saúde.

\section{Discussão}

O presente estudo evidenciou que os indicadores relacionados a estrutura e processo nas instituições hospitalares estudadas apresentaram, de maneira geral, média de conformidade abaixo do esperado, correspondente a $100 \%$. Foi observado que entre os hospitais há necessidades que foram comuns à maioria das instituições avaliadas como a indisponibilidade de espaços físicos exclusivos para as atividades; realização de reuniões periódicas com lideranças e membros da CCIH; padronização de soluções germicidas e antissépticos; elaboração de relatórios com análise dos resultados das infecções hospitalares; e realização de busca ativa dos casos de IRAS e visitas de inspeção.

Segundo a portaria 2616/98, compete à Coordenação Municipal de Controle de IRAS coordenar as ações de prevenção e controle de IRAS na rede hospitalar dos municípios de forma a colaborar e acompanhar os hospitais na execução destas ações; e prestar apoio técnico às comissões dos hospitais (Brasil, 1998). O não atendimento destas normativas leva a discussão de que há um hiato entre a teoria e a prática nas atividades desenvolvidas, principalmente devido à falta de fiscalização por conta dos órgãos competentes, para que os profissionais da CCIH e da assistência assumam o compromisso de implementar as práticas de controle destas infecções em suas rotinas. Esta realidade também seria diferente se os hospitais em estudo fossem acreditados com certificação concedida pela Organização Nacional de Acreditação (ONA) uma vez que para isso seria necessário o cumprimento de critérios de qualidade nos serviços oferecidos, e o PCIRAS é um importante 
controlador da qualidade da assistência à saúde (Gomes \& Gomes, 2018).

A média de conformidade geral observada nos hospitais avaliados estão próximas das observadas em estudos realizados em hospitais de Ribeirão Preto, 68,8\%; Campo Grande, 71,5\% e Paraná, 71,0\% (Menegueti et al., 2015; Giroti et al., 2018; Alves \& Lacerda, 2015). Isto demonstra que as práticas de controle de infecção nos hospitais municipais de São Luís não diferem muito de hospitais de outras regiões do país. Assim, torna-se importante que os componentes de menor conformidade sejam revistos e implementadas ações que levem ao aprimoramento dos processos deficientes, uma vez que o ideal seria alcançarem $100 \%$ de conformidade.

Pode-se observar também que não há padronização nas rotinas implantadas nos hospitais, pois em cada indicador houve variações significativas entre os itens avaliados, alguns deles com resultados excelentes, com $100 \%$ de conformidade e outros, muito aquém do ideal. O indicador Estrutura Técnico-Operacional do PCIRAS, evidenciou que apenas 37,5\% das instituições possuem espaço físico exclusivo para suas atividades, semelhante ao apresentado no estudo realizado em Ribeirão Preto (30,0\%) (Menegueti et al., 2015). Pode-se sugerir que isto aconteça porque o gestor da unidade desconhece a legislação pertinente, que obriga a autoridade máxima da instituição propiciar a infraestrutura necessária à correta operacionalização do PCIRAS (Brasil, 1998), ou, porque a área física do hospital é inapropriada, e não há espaço suficiente para deixar uma área exclusiva para este serviço e para os núcleos hospitalares, também obrigatórios, conforme legislação brasileira.

Outro componente com menor conformidade se refere a reuniões com lideranças e membros executivos, que devem ser realizadas ordinariamente, uma vez a cada mês. De acordo com Santos et al. (2017), reuniões periódicas devem ser realizadas com o objetivo de aprimorar as práticas de controle de infecção hospitalar; permitindo que haja troca de saberes e opiniões, de forma interdisciplinar, permitindo que profissionais com formações diferentes exponham suas opiniões sobre as questões abordadas e se responsabilizem pelas ações a serem adotadas. A realização destas reuniões é de grande importância para implementação das práticas de controle de infecção, pois com a participação de diferentes profissionais, inclusive gestores da unidade, se consegue, em pouco tempo, ter acesso aos problemas pertinentes a cada setor; além de incitar esses profissionais a sugerirem estratégias a serem desenvolvidas.

O indicador Sistema de Vigilância Epidemiológica das IRAS alcançou índice de 55,7\%, enquanto nos municípios de Ribeirão Preto e Campo Grande, os resultados foram bem superiores: 83,7\% e 82,0\%, respectivamente (Menegueti et al., 2015; Giroti et al., 2018). Este baixo índice pode estar relacionado à falta de conhecimento dos profissionais quanto à importância de realizar a vigilância epidemiológica das IRAS para identificar o perfil das infecções constantes na unidade, os fatores que interferem no controle das IRAS, e definir estratégias de enfrentamento a serem adotadas.

Foi constatado que os relatórios periódicos com resultados das IRAS são realizados somente em 50,0\% das unidades avaliadas. A CCIH pode utilizar relatórios para transmitir informações relevantes e compartilhar resultados, levando a elaboração de planos de ações que possam servir de ajuda aos setores pertinentes (Campos et al., 2019). A não realização destes relatórios pode estar relacionada a falta de sistematização das atividades desenvolvidas pelos controladores de infecção; número insuficiente de profissionais para realização das atividades; demanda de atividades não relacionadas ao controle de infecções que acabam sendo impostas para os membros da $\mathrm{CCIH}$; inclusão destes profissionais em outro setor; falta de capacitação da equipe e falta de supervisão de gestores e órgãos competentes.

Quanto à realização de busca ativa de infecções hospitalares, imprescindível para ser realizado vigilância epidemiológica das IRAS, foi constatado que esta prática é rotina nas quatro unidades que possuem UTI; entretanto, nas outras unidades, apenas uma realiza esta atividade. As notificações das IRAS são obrigatórias para os chamados serviços prioritários (hospital com leitos de UTI, Centro Cirúrgico, Centro Obstétrico e Serviços de Diálise que prestam assistência a pacientes crônicos) (Anvisa, 2021). Talvez a não obrigatoriedade de fazê-lo em setores de baixo risco, como as enfermarias de Clínica Médica e Cirúrgica, faz com que os profissionais acabem por priorizar outras atividades desenvolvidas pelo setor. Com esta 
atitude, deixam de identificar sinais precoces de IRAS, e de intervir quando necessário, o que poderia evitar a disseminação destas infecções entre os pacientes hospitalizados.

O indicador que avalia a realização de atividades de controle e prevenção de IRAS nos diversos setores do hospital, apresentou menor média de conformidade geral de 44,9\%. Observou-se que nos setores onde há presença de pacientes internados como UTI e enfermarias, houve $100 \%$ de conformidade. Entretanto, apenas $16,7 \%$ das unidades avaliadas realizam estas atividades nos ambulatórios, 14,3\% nos setores de Pronto Socorro e 12,5\% no Serviço de Nutrição e Dietética. Cabe ressaltar, que atividades de controle e prevenção de IRAS com muito baixa conformidade, ou mesmo nula, podem comprometer a qualidade das atividades fins, e não isenta a responsabilidade dos profissionais da CCIH (Alves \& Lacerda, 2015).

O indicador que avalia as diretrizes operacionais de controle e prevenção de IRAS apontou para a presença de padronização de soluções germicidas e antissépticos em apenas 37,5\% das unidades avaliadas. Este item também apresentou valor insatisfatório nos estudos realizados no Paraná (54,0\%) e Ribeirão Preto (53,8\%) (Alves \& Lacerda, 2015; Menegueti et al., 2015).

A utilização sem critérios de soluções germicidas e antissépticos pode prejudicar a técnica de higienização das mãos e levar a falhas nos processos de limpeza e desinfecção de materiais, equipamentos e superfícies. Segundo a Anvisa (2010), o aparecimento de infecção hospitalar pode estar relacionado a falhas nos processos de limpeza e desinfecção de superfícies, que podem ter como consequência a disseminação e transferência de microrganismos nos ambientes dos serviços de saúde, colocando em risco a segurança dos pacientes e dos profissionais que atuam nesses serviços.

Os profissionais da CCIH devem conhecer todos os saneantes e antissépticos utilizados na unidade e caso algum destes não obedeça aos critérios definidos pela legislação, podem indeferir seu uso. As recomendações de diluição, armazenamento, utilização e destinação final destes produtos também devem ser aprovados pela CCIH e cabe a esta realizar fiscalização contínua para que todas as etapas sejam realizadas adequadamente.

A responsabilidade na seleção, escolha e aquisição dos produtos saneantes deve ser da CCIH, conjuntamente com o Serviço de Limpeza e com o Setor de Compras da instituição (Anvisa, 2010). No entanto, em apenas uma das unidades avaliadas, a CCIH tem a possibilidade de participar nas decisões técnicas para especificação e aquisição de produtos e correlatos. Nas demais, cabe aos controladores de infecção analisarem a qualidade dos produtos a serem utilizados, e quando inadequados, têm que enfrentar uma série de burocracia para impedir seu uso.

A presença de recomendação de técnica de higienização das mãos esteve presente em $87,5 \%$ das unidades. No entanto, almejava-se que obtivesse o valor máximo na pesquisa, por ser amplamente reconhecida como uma das principais estratégias para a prevenção das IRAS (Price et al., 2018; Anvisa, 2018).

A higienização das mãos, assim como a adoção de outras medidas preventivas no ambiente hospitalar, como o uso de equipamentos de proteção individual, nunca foi tão difundida, como na atual pandemia de Covid-19. Nunca se vivenciou com tanta intensidade a necessidade de práticas de controle de infecção implantadas de forma efetiva. Quantas mortes teriam sido evitadas, principalmente entre os profissionais de saúde, se estas práticas já fossem realidade na rotina das instituições. Observou-se que nas unidades avaliadas, principalmente nas que se tornaram referências para atendimento a pacientes com Covid-19, houve uma evolução considerável, serviços foram informatizados, documentos (manuais, procedimentos operacionais padrões, regimentos, portarias, entre outros) foram elaborados, núcleos de vigilância foram constituídos, e o serviço de controle de infecção hospitalar passou a ser mais reconhecido pela sua real importância, o qual, para muitos, era visto como um serviço dispensável.

No entanto, é preciso que as diretrizes instituídas sejam efetivamente operacionalizadas, que os controladores de infecção continuem aperfeiçoando suas ações para melhorar os indicadores de qualidade da assistência prestada aos pacientes, 
e para tanto, que os hospitais utilizem os resultados obtidos em pesquisas para intervir diretamente nos pontos em que precisam ser melhorados para qualificar seus serviços. Este estudo trata-se da primeira avaliação desta magnitude realizada no município de São Luís e pode ser utilizado para impactar na melhoria dos indicadores de controle de infecção dos hospitais avaliados.

\section{Conclusão}

Os serviços avaliados apresentaram melhor desempenho nos indicadores de estrutura e denotam fragilidade quanto às atividades essenciais para prevenção de infecções hospitalares, como realização de vigilância epidemiológica e desenvolvimento de estratégias de prevenção e controle destas infecções em diversos setores do hospital. No entanto, todos os indicadores avaliados apresentaram deficiência em seus componentes, pois mostraram índices de conformidade inferior ao que seria almejado, retratando deficiências nas medidas de controle e prevenção de IRAS.

Vale considerar também que os baixos índices de conformidade podem ter sido influenciados pela falta de conhecimento de alguns profissionais entrevistados quanto à alguns itens abordados no instrumento, uma vez que foi observado em alguns serviços, que devido à pandemia, profissionais que atuavam na CCIH precisaram ser afastados e foram substituídos por profissionais sem qualquer experiência na área. O que poderia ter sido avaliado se o estudo incluísse um segundo instrumento, contendo dados referentes ao perfil dos membros executores da CCIH e também da instituição avaliada.

A pandemia trouxe outros percalços que influenciaram no estudo, como: limitação de acesso às unidades, o que retardou o início da coleta e o medo da contaminação pelo covid-19 tanto por parte do pesquisador como também dos profissionais entrevistados; ainda assim todos concordaram em participar da pesquisa.

Sugere-se que os indicadores utilizados neste estudo sejam utilizados posteriormente para novas avaliações a serem realizadas nestas instituições, e ainda que esta pesquisa seja ampliada para outros hospitais públicos e privados de São Luís e de outros municípios, com vistas a possibilitar uma melhoria da qualidade da assistência à saúde da população.

\section{Agradecimentos}

Gostaríamos de agradecer à Secretaria Municipal de Saúde por permitir a realização da pesquisa em suas unidades de saúde, e aos membros da CCIH que dedicaram um pouco do seu precioso tempo para colaborar com a coleta dos dados.

\section{Referências}

Agência Nacional de Vigilância Sanitária. (2010). Segurança do paciente em serviços de saúde: limpeza e desinfecção de superfícies. Brasília (DF): Anvisa.

Agência Nacional de Vigilância Sanitária. (2018). Nota Técnica No01/2018 GVIMS/GGTES/ANVISA: orientações gerais para higiene das mãos em serviços de saúde. Brasília (DF): Anvisa.

Agência Nacional de Vigilância Sanitária. (2021). Iras e resistência microbiana: formulários para notificação. https://www.gov.br/anvisa/ptbr/assuntos/noticias-anvisa/2021/iras-e-resistencia-microbiana-formularios-para-notificacao.

Alves, D. C. I., \& Lacerda, R. A. (2015). Avaliação de programas de controle de infecção relacionada a assistência à saúde de hospitais. Rev Esc Enferm USP., 49(Esp), 65-73. DOI: 10.1590/S0080-623420150000700010.

Bordignon, R. P., Schuh, L. X., Cremonese, L., Merenhque, C. L., Fagundes, P. T., \& Barreto, C. N. (2020). Saberes e práticas de enfermeiros intensivistas no controle da infecção hospitalar. Research, Society and Development, 9(7), 1-17, e327974094.

Brasil. (1997). Ministério da Saúde. Lei $n^{\circ}$ 9.431, de 06 de janeiro de 1997. Dispõe sobre a obrigatoriedade da manutenção de Programas de Controle de Infecções Hospitalares nos hospitais do país. Brasília (DF): Diário Oficial da União.

Brasil. (1998). Ministério da Saúde. Portaria $n^{\circ}$ 2.616, de 12 de maio de 1998. Dispõe sobre diretrizes e normas para a prevenção e o controle das infecções hospitalares. Brasília (DF): Diário Oficial da União.

Campos, C. E. M., Canto, D. F., Aldabe, L. M., Costa, F. M., \& Chaves, E. H. B. (2019). Relatórios informativos e boas práticas de enfermagem: um relato de experiência. Anais da $39^{\circ}$ Semana Científica do Hospital de Clínicas de Porto Alegre, Porto Alegre, RS.

Costa, M. M. M. (2016). Efeitos de um ciclo de melhoria da qualidade nacional aplicado à estruturação das ações de prevenção das infecções relacionadas à assistência à saúde em hospitais brasileiros [dissertação de mestrado]. Universidade Federal do Rio Grande do Norte, Natal, RN. 
Giroti, A. L. B., Ferreira, A. M., Rigotti, M. A., Sousa, A. F. L., Frota, O. P., \& Andrade, D. (2018). Hospital infection control programs: assessment of process and structure indicators. Rev Esc Enferm USP, 52, e03364. DOI: http://dx.doi.org/10.1590/S1980-220X2017039903364

Gomes, M. F., \& Gomes, V. L. (2018). O programa de controle de infecção relacionada à assistência à saúde em meio ambiente hospitalar e o dever de fiscalização da Agência Nacional de Vigilância Sanitária. R. Dir. sanit., 18(3), 43-61.

Instituto Brasileiro de Geografia e Estatística (2010). Censo Brasileiro de 2010. https://www.ibge.gov.br/cidades-e-estados/ma/sao-luis.html.

Instituto Latino Americano de Sepse. (2016). Sepse: um problema de saúde pública. Brasília (DF): Conselho Federal de Medicina.

Menegueti, M. G., Canini, S. R. M. S., Bellissimo-Rodrigues, F., \& Laus, A. M. (2015). Evaluation of nosocomial infection control programs in health services. Rev Latino Am Enfermagem, 23(1), 98-105.

Oliveira, H. M., Silva, C. P. R., \& Lacerda, R. A. (2016). Políticas de controle e prevenção de infecções relacionadas à assistência à saúde no Brasil: análise conceitual. Rev. da Escola de Enfermagem da USP, 50(3), 505-11.

Price, L., Melonea, L., Mclarnona, N., Bunyan, D., Kilpatrick, C., Flowersa, P., \& Reillya, J. (2018). A systematic review to evaluate the evidence base for the World Health Organization's adopted Hand Hygiene Technique for reducing the microbial load on the hands of Healthca workers. American Journal of Infection Control, 46, 814-23.

Santos, E. O., Coimbra, V. C. C., Kantorski, L. P., Pinho, L. B., Andrade, A. P. M., \& Eslabão, A. D. (2017). Reunião de equipe: proposta de organização do processo de trabalho. Rev Fund Care Online, 9(3), 606-613. DOI: http://dx.doi.org/10.9789/2175-5361.2017.v9i3.606-613.

Schmitt, C. (2015). Características e iniciativas institucionais que aprimoram as práticas de uso de antibioticoprofilaxia cirúrgica [tese de doutorado]. Escola de Enfermagem, Universidade de São Paulo, São Paulo, SP.

Siegel, J. D., Rhinehart, E., Jackson, M., Chiarello, L., \& Healthcare Infection Control Practices Advisory Committee (2007). 2007 Guideline for isolation precautions: preventing transmission of infectious agents in healthcare. Am J Infect Control., 35(10), 65-164. https://dx.doi.org/10.1016/j.ajic.2007.10.007.

Silva, C. P. R., \& Lacerda, R. A. (2007). Indicadores para avaliação de programas de controle de infecção hospitalar: construção e validação. Epidemiol Serv Saúde, 16(2), 128-31.

Storr, J., Twyman, A., Zingg, W., Damani, N., Kilpatrick, C., Reilly, J. ... \& WHO Guidelines Development Group. (2017). Core components for effective infection prevention and control programmes: new WHO evidence-based recommendations. Antimicrob Resist Infect Control., 6, 6. doi: 10.1186/s13756-0160149-9. PMID: 28078082; PMCID: PMC5223492.

World Health Organization. (2014). Health care-associated infections Fact Sheet. Genebra: WHO. 